\title{
Hepatic congestion is the main pathological sign in concanavalin A-induced acute hepatitis in Bama mini-swine.
}

\author{
Zongzong Quan, Guifang Yu*, Boxiong Shen, Shanliang Jin
}

Department of Anesthesiology, Shanghai Ninth People's Hospital, School of Medicine, Shanghai Jiaotong University, Shanghai 201999, PR China

\begin{abstract}
Objective: The plant lectin concanavalin A (ConA) induced acute hepatitis in mice is a commonly used animal model applied in acute immune-mediated hepatitis studies, but it is not suitable for large animal model of hepatic injury to promote the study of bio-artificial liver support systems in humans and help to assist pre-clinical evaluation. During producing Bama mini-swine model of hepatic injury, we find Hepatic congestion is dispensable for this model.

Methods: Seven Obama mini-swines in the model group were exposed to ConA by intravenous injection $(5 \mathrm{mg} / \mathrm{kg})$ into the vein of auricular back. The other 3 miniswines in the control group was administered with equal volume of saline. Blood was collected from precaval vein at $0 \mathrm{~h}, 6 \mathrm{~h}$ and $24 \mathrm{~h}$ after injection. Serum alanine aminotransferase (ALT), aspartate aminotransferase (AST), serum creatinine (SCr) were determined. Portal vein pressure was measured. Animals surviving $24 \mathrm{~h}$ were euthanased. Afterwards, Liver tissues were collected and liver/weight index was calculated. The histological analyses of liver, kidney and spleen were performed.

Results: Our data show that hepatic congestion is the main pathological sign in concanavalin A-induced acute hepatitis model in mini-swine resulting in hepatitis congestion, intestinal bleeding, marked renal tubular necrosis and spleen.

Conclusion: Hepatic congestion is the main pathological sign in concanavalin A-induced acute hepatitis in Bama mini-swine.
\end{abstract}

Keywords: Hepatic congestion, Concanavalin A, Hepatitis, Bama mini-swine.

Accepted on May 23, 2017

\section{Introduction}

Liver the largest internal organ in human converts food nutrients into enzymes, vitamins, produces bile which is required for digestion and breaks down substances like alcohol. There are many factors such as Virus infection, autoimmune conditions, alcohol intake, and the use of certain drugs can cause hepatitis and finally develop in liver cirrhosis, liver failure or liver cancer easily, Although there are many ways to deal with it, which may promote the recovery of liver function, but unfortunately there are still a large number of patients have no response to treatment, and even in company with secondary damage to other organ function or further deterioration of liver function, eventually need for liver transplantation. Liver transplant involves transplantation of liver from a person deceased recently or a part of liver taken from a living person or donor. As liver has regeneration property, both the patient's and donor's liver grows and becomes fully functional in matter of weeks. The possibility of liver transplant was started in late 1960 s and with the advance in surgery techniques and improved immune-suppressants to reduce the rejection rates, the success rate of liver transplantation is high.
The most common reason for failure of liver is due to hepatitis virus. The hepatitis pathogen causes chronic infection in human liver and millions worldwide are affected. Various researches focus on the biology and treatment of hepatitis. Animal model which support hepatitis pathogen is required for the research. Extensive studies where mice, chimpanzees, pigs are used for the study of liver failure and its treatment. The animal models are infected to cause the hepatitis and subsequent testing is carried out. It is known that Con Ainduced acute hepatitis resembles fulminant hepatitis (FH) in many aspects [1], such as severe acute liver failure, massive hepatocellular degeneration, infiltration of lymphocytes in liver, activation of $\mathrm{T}$ and natural killer (NK) cells [2]. Therefore, Con A-induced FH in mice greatly mimics the immune and inflammatory response of $\mathrm{FH}$ in humans and is a good model to well simulate human viral hepatitis, autoimmune hepatitis, and the pathophysiology of acute liver failure.

Hepatitis causes liver fibrosis which an over build-up of extracellular matrix such as collagen leading to cirrhosis, liver failure, and portal hypertension. Advanced hepatic fibrosis will often require liver transplantation. Our hypothesis was that 
Portal hypertension is the initiating factor of acute liver injury, rather than the terminal point of liver injury.

\section{Materials and Methods}

\section{Animals}

Ten 2-year-old 12 to $30 \mathrm{~kg}$ male Bama mini pigs (Shanghai, China). were boarded in separate wire-rod-floored, stainlesssteel cages of dimension $100 \times 100 \times 100 \mathrm{~cm}$, with a collection pan underneath each cage and a temperature at 20 to $22^{\circ} \mathrm{C}$, relative humidity of $50 \%$ to $55 \%, 10$ to 15 air changes per hour, $12 \mathrm{~h}$ day/night shift, unlimited water supply, and were fed on a standard swine diet (500 g daily; Lab swine Chow, Shanghai, China). In the procedure room, the temperature was 20 to 22 . The Bama mini pigs were isolated 15 days earlier to adapt to environmental conditions, food, and water to allow daily assessment of health condition. Treatment order was randomized, and each mini pig received all treatments with at least 30 days between experiments. All the experiments involving animal were carried out in accordance with the Home office regulations under the Animal (Scientific procedures) Act 1986 as per project license 60/2389. All animals received humane care and the institution's guidelines on study protocols were observed. All animals received humane care according to the guidelines established by the National Science Council of the People's Republic of China.

Anesthesia was induced with $\mathrm{Su}$ Mian Xinwhich was purchased from Sheng Da Animal Drug Co. Ltd., (Dun Hua, Ji LIN, China) and successively sustained with isoflurane according to tidal volume. Background hydration combined with $0.9 \%$ Normal Saline and 5\% Dextrose was continued at a rate of $2 \mathrm{ml} / \mathrm{kg} / \mathrm{h}$ as per electrolyte results from arterial blood gas sampling and urine output. To correct the hypotension, the Boluses of colloid (Gelofusine) were injected. The core (rectal) temperature of $37-39^{\circ} \mathrm{C}$ was maintained using heater pads. The Haemodynamic variables was checked constantly by Datex AS/3 monitoring system (Datex Ohmeda, Stirling, UK) and was documented at five minute intervals by using the Datex Collect Programme on a laptop computer. Pressure of airway (PAW), blood pressure (BP), Pulse (P), $\mathrm{SPO}_{2}$ was continuously recorded during anesthesia.

\section{Experimental design}

On the day of each experiment, each Bama mini pig was weighed $1 \mathrm{~h}$ before physiologic variables were measured. Conscious Bama mini pigs were placed into restraining cages for catheter insertion and during the intramuscular injection for induction of anesthesia. A 24-gauge intravenous catheter was placed in the marginal ear vein under local anesthesia, which was applied to the ear 45 to 60 min before concanavalin A was given.

After adaptive breeding for one week, in the model group, 5 $\mathrm{mg} / \mathrm{kg}$ ConA was introduced into the vein on ear back. The swine in the control group was given equal volume of saline with the dose.

\section{Concanavalin}

A administration. Concanavalin A was purchased from Sigma Aldrich (St. Louis, MO) and it was dissolved in sodium chloride. In seven animals, intravenous Concanavalin A was injected while three animals were used as controls and monitored invasively but did not receive any Concanavalin A infusions.

Determination of ALT, AST and SCr. Standard laboratory assays were used for the measurement of liver and renal function, Serum aspartate aminotransferase, albumin, and creatinine analysis were measured on an Olympus AU2700 automated analyser (Olympus UK Ltd, Watford, UK.) using proprietary diagnostic kits.

Taking of Photos of the liver and intestines after the end of modeling, the swine was laparotomize during anesthesia. The liver and the intestine photos were taken by an OLYMPUS c-120 digital camera which was $30 \mathrm{~cm}$ away from the liver and the intestines under a standard light source (color temperature 5300).

Direct assessment of Portal Vein Pressure The portal vein pressure was assessed directly during laparotomy. After exposure of portal vein, a 16-gauge intravenous cather (Arrow International Inc, PA, USA) was placed in the portal vein. Intravenous transducer (Smith medical called Single Monitoring Kit, Dublin, USA) was used to assess the poral vein pressure.

Tissue Preparation and HE staining Experiments lasted up to $24 \mathrm{~h}$, and animals surviving at this timepoint were euthanized. Liver of each Bama mini pig was weighed and then calculated the index of liver/weight. The organs designed for morphological analysis (liver, kidney, and spleen) were quickly removed, rinsed with saline solution $(0.9 \%)$, and fixed in formalin $10 \%$. The diagonal section of the liver and spleen as well as the longitudinal section of the kidney was obtained and processed (Pathology laboratory, pathology department of Shanghai Jiaotong university affiliated NO.3 hospital). The processed tissues were embedded in paraffin, sectioned at $4 \mathrm{~m}$ thickness, and placed on frosted glass slides for further evaluation. The tissue macroscopic alterations were also analyzed.

The samples were stained using hematoxylin and eosin (H\&E) stains, which can detect changes in the nucleus and cytoplasm; Hematoxylin and eosin (H\&E) staining were acquired from pathology department of Shanghai Jiaotong university affiliated NO.3 hospital. The slides were assessed using a light microscopy coupled to the photomicrographic camera, both adapted to a micro-computer with software Honestech for image capture.

\section{Statistical analysis}

Statistical analysis was performed by using commercially available computer software (GraphPad Prism 5). The parametric results are expressed as mean \pm SD. Differences 
were considered significant when the $\mathrm{P}$ value was less than 0.05 .

\section{Results}

\section{Changes of haemodynamic variables}

Table 1 establishes an increase in pulse and concomitant reduction in BP. The injured pigs (compared to uninjured pigs) showed the typical haemodynamic pattern of acute liver injury characterized by arterial hypotension $(48.9 \pm 4.3$ vs. $60.3 \pm 2.1$ $\mathrm{mmHg}, \mathrm{p}=0.16$ ) (MAP, mean arterial pressure). Positive pressure lung ventilation was accompanied with a decline in total compliance as injury progressed and the Pressure of airway (PAW) $\left(13.7 \pm 1.5\right.$ vs. $\left.18.6 \pm 2 \mathrm{~cm} \mathrm{H}_{2} \mathrm{O}, \mathrm{p}<0.05\right)$ increased in this group (Table 1). This may have point to the beginning of an adult respiratory distress syndrome (ARDS). During the maintenance of anesthesia in groups, $\mathrm{SpO}_{2}$ remained similar $(\mathrm{P}>0.05)$ in both groups.

Table 1. Influence of ConA injection on Haemodynamic variable $($ mean \pm SEM) in Bama mini-pig 24 h later.

\begin{tabular}{lllll}
\hline & MAO $(\mathbf{m m H g})$ & $\mathrm{SPO}_{2}(\%)$ & PAW $\left(\mathbf{c m H}_{2} \mathbf{O}\right)$ & Pulse \\
\hline $\begin{array}{l}\text { Control group } \\
(\mathrm{n}=3)\end{array}$ & $60.3 \pm 2.1$ & $99 \% \pm 1 \%$ & $13.7 \pm 1.5$ & $74.0 \pm 12$ \\
\hline $\begin{array}{l}\text { Injured group } \\
(\mathrm{n}=7)\end{array}$ & $48.9 \pm 4.3^{\mathrm{a}}$ & $\begin{array}{l}97.7 \% \\
\pm 1.1 \% \mathrm{~b}\end{array}$ & $18.6 \pm 2.0^{\mathrm{a}}$ & $103.9 \pm 9.6^{\mathrm{a}}$ \\
\hline
\end{tabular}

MAP: Mean Arterial Pressure; PAW: Pressure of Airway; ${ }^{\mathrm{a}} \mathrm{p}<0.05 ;{ }^{\mathrm{b}} \mathrm{P}>0.05$

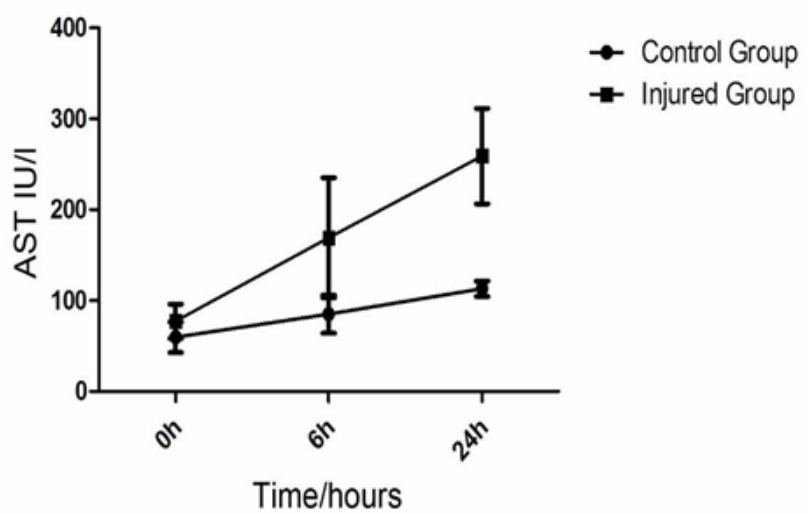

A

Figure 1. Profile of serum AST in control and ConA injured swines. It depicts the increase in serum AST at 3 different time.

Comparison of Liver Functions and Renal Functions between the Injured Swine and the Normal Swine Biochemical analysis revealed that AST and ALT levels were equivalent between two groups at the (baseline) (0-min time point). But at $6 \mathrm{~h}$ and $24 \mathrm{~h}$ after ConA injection, a marked increase in serum AST $(231 \pm 76.7$ vs. $91 \pm 15.7, \mathrm{p}=0.03)$ in injured group (Figure 1). AST rather than ALT was used to quantify liver damage as the assay employed which was unable to measure porcine ALT from homogenised porcine liver tissue. In addition to liver injury there was evidence of renal impairment as also shown in
Figure 2 that serum creatinine rises above $90 \mu \mathrm{mol} / \mathrm{L}$ and the mean levels of creatinine were199 \pm 85 in injured animals $(\mathrm{p}=0.01)$.

\section{Change of microvascular blood flow perfusion in the liver and intestines}

The liver and intestines color in the injured mini-swine show connection (Figure 3).

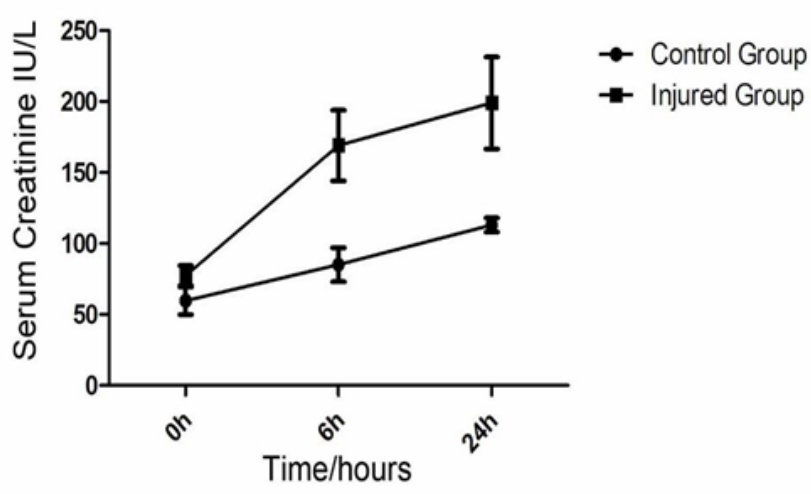

B

Figure 2. Profile of serum Creatinine in control and ConA injured swines. It depects the increse in serum Creatinine at 3 different time.

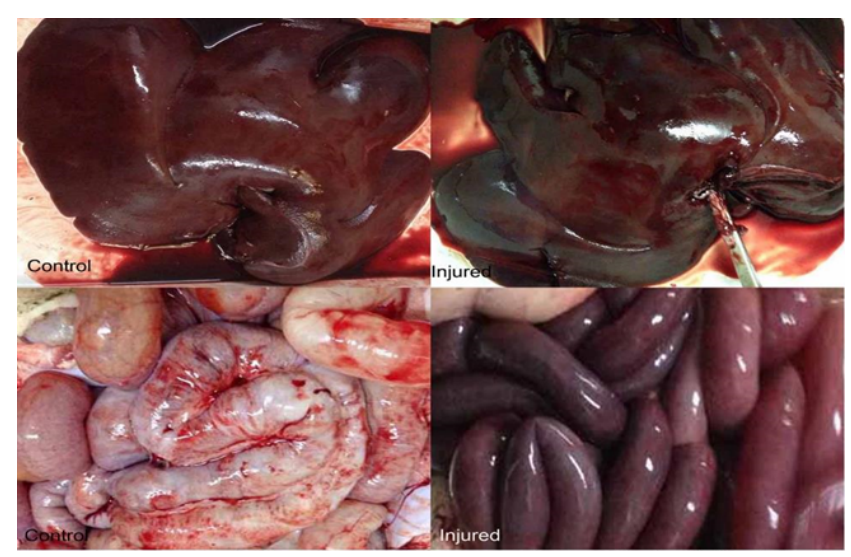

Figure 3. Liver and intestine of mini-swine exposed to or not ConA; The picture is a representation of three independent experiments in 5 $\mathrm{mg} / \mathrm{kg}$ ConA tested after $24 \mathrm{~h}$.

\section{Change of portal vein pressure}

$24 \mathrm{~h}$ after ConA injection, Portal Vein Pressure measured from the injured swine significantly increased. The portal vein pressure was $6.333 \pm 2.028 \mathrm{mmHg}$ in the normal swine and $22.00 \pm 2.895 \mathrm{mmHg}$ in the swine with liver injury, showing significant difference between the two groups, $\mathrm{P}<0.05$.

\section{Change of histology}

Histological analysis of the pig liver tissue showed perivenular and midzone confluent hepatocytic necrosis in the badlyaffected animals, with micro-vesicular changes in preserved viable parenchyma. Other animal models had lesser injury based on the degree of necrosis, but qualitatively comparable 
patterns were seen. Of the test animals, 4 animals had moderate severely affected liver injury, 2 animals had severe coagulative necrosis and 3 animals had mild no zonal confluent necrosis. Correspondingly, renal histopathology of 7 pigs showed severe acute tubular injury based on the prevalence of vascular change to the cortical tubular epithelium.

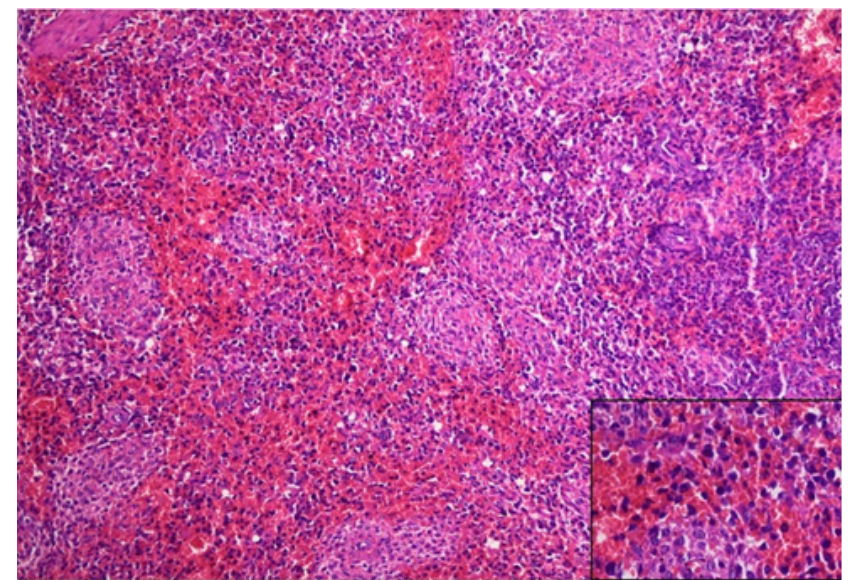

Figure 4A. Spleen histological analysis of mini-pig exposed $5 \mathrm{mg} / \mathrm{kg}$ ConA 24 h later. $H \& E$ stained section demonstrating prominent red flares around a group of highly reactive white pulp nodules, representing peri-folliculer red pulp intensely connected with red blood cells.

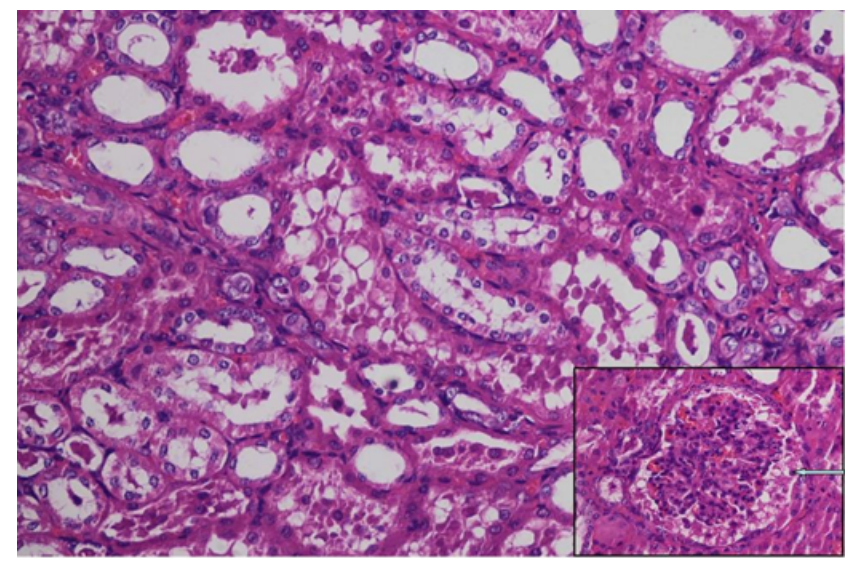

Figure 4B. Renal histological analysis of mini-pig exposed $5 \mathrm{mg} / \mathrm{kg}$ ConA 24 h later. H\&E stained section demonstrating renal injury (10X). Arrow means renal injury (detail 40X).

In the normal liver tissues, the hepatic cells organized in correct order with no inflammation; though propagation of a few fibers in the portal area, lymphocyte infiltration, proliferation of epithelium of biliary duct, and acidophilic necrosis was seen in liver injury tissue.

\section{Discussion}

The pathophysiology of AHF is an active research area. It is apparent that there is an existing relationship between various pathogenic factors, such as bacteria toxins, cytokines, free radicals and other constituents of the inflammatory system causing the local lesions [3]. It has been observed that initially endothelium releases vasoactive agents affecting the local and distal blood flow in the dire phase of the disease, with nitric oxide, prostacyclins and endothelins being important components of the response [4]. Concanavalin A (ConA) is a powerful inducer of inflammatory cytokines from the innate immune cells. Intravenous administration of this lectin outcomes in progressive hepatic inflammation in mice $[5,6]$. The inflammatory effects are visible within $6 \mathrm{~h}$ of ConA treatment, with attachment of leukocytes to the hepatic endothelium and blood cells flow out of the centre vein. In about $24 \mathrm{~h}$, lymphocytes sticks to hepatocytes. Thus, it is seen that the ConA-induced hepatic injury leads to deleterious effects of inflammatory molecules given out by a number of cellular sources, including Kupffer cells, NK cells, leukocytes, and endothelial cells (Figures 4A-4C).

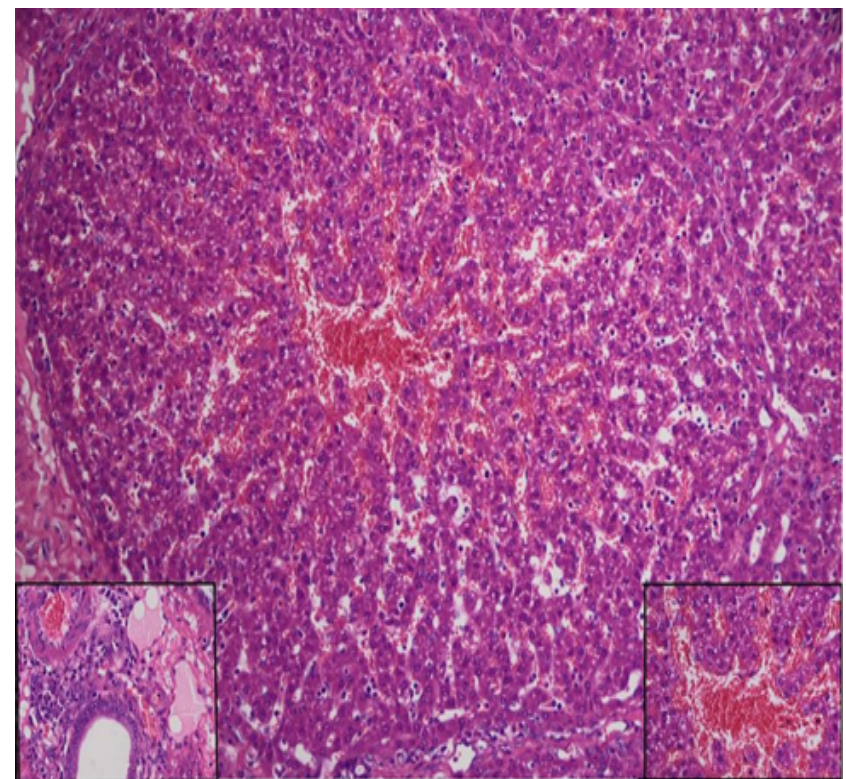

Figure 4C. Liver histological analysis of mini-pig exposed $5 \mathrm{mg} / \mathrm{kg}$ ConA 24 h later. $H \& E$ stained section demonstrating monster cells with normal nuclei, dispersed chromatin and nucleolus arranged towards hepatic central vein which were connected with red blood cells (details in 40X), there also .

Changes of Blood Flow Perfusion in liver tissue during the course of ConA-induced hepatitis have not been previously studied. It is unknown whether the portal pressure induced renal low perfusion which results in severe acute tubular injury. In this assay a low dose of ConA $(5 \mathrm{mg} / \mathrm{kg})$ was employed. The progressive increases in serum ALT, AST and Scr. Inexplicably, however, the photo of the liver and intestine changes $24 \mathrm{~h}$ after injection with dark color appearing (Figure 3).

\section{Limitations}

The present study has few limitations. First, it is an animal study and with a small sample. Second, all of the observations during the experiments are made by the physicians are selfreported and self-judgment; therefore, the results cannot be generalized to all KAUH doctors. Additionally, the study was conducted in one center, so results cannot be generalized. 


\section{Conclusion}

In conclusion, the results of the current studies show high portal vein pressure is the initiating factor of acute liver injury, which occurs early in the course of disease $3 \mathrm{~h}$ after $5 \mathrm{mg} / \mathrm{kg}$ ConA injection.

\section{Acknowledgement}

Grant Support \& Financial Disclosures: Xinhua Group Foundation. Zongzong Quan: Designed the study, conducted data analysis and manuscript writing. Guifang Yu: Conceived the idea, supervised the data collection, helped in data analysis and manuscript writing. Shangliang Jin and Boxiong Shen: Conducted the fieldwork and data entry on SPSS, helped in analysis and interpretation of data, writing the final manuscript.

\section{References}

1. Miyazawa Y. In development of intrasinusoidal hemostasis in the development of concanavalin A inducedhepatic injury in mice. Hepatology 1998; 27: 497-506.

2. Tiegs G. A T cell-dependent experimental liver injury in mice inducible by concanavalin A. J Clin Invest 1992; 90 : 196-203.
3. Leifeld L, Dumoulin FL, Purr I, Janberg K, Trautwein C, Wolff M, Manns MP, Sauerbruch T, Spengler U. Early upregulation of chemokine expression in fulminant hepatic failure. J Pathol 2003; 199: 335-344.

4. Zwingmann C, Chatauret N, Leibfritz D, Butterworth RF. Selective increase of brain lactate synthesis in experimental acute liver failure: results of a [H-C] nuclear magnetic resonance study. Hepatology 2003; 37: 420-428.

5. Tiegs G, Hentschel J, Wendel JA. T-Cell-dependent experimental liver injury in mice inducible by concanavalin A. J Clin Invest 1992; 90: 196-203.

6. Tiegs G. Experimental hepatitis and the role of cytokines. ActaGastroenterol Belg 1997; 60: 176-179.

\section{*Correspondence to}

Guifang $\mathrm{Yu}$

Department of Anesthesiology

Shanghai Ninth People's Hospital

School of Medicine

Shanghai Jiaotong University

PR China 